\title{
Evolution of the radial electric field in a JET $\mathrm{H}$-mode plasma
}

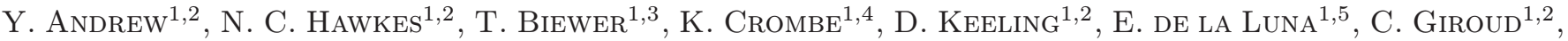 \\ A. Korotkov ${ }^{1,2}$, A. Meigs ${ }^{1,2}$, A. Murari ${ }^{1,6}$, I. Nunes ${ }^{1,7}$, R. Sartori ${ }^{1,8}$, T. TAla ${ }^{1,9}$ and JET-EFDA \\ CONTRIBUTORS $^{1(a)}$ \\ 1 JET-EFDA, Culham Science Centre - Abingdon, OX14 3DB, UK, EU \\ ${ }^{2}$ Euratom/UKAEA Fusion Association, Culham Science Centre - Abingdon, OX14 3DB, UK, EU \\ ${ }^{3}$ Oak Ridge National Laboratory - Oak Ridge, TN 37831, USA \\ ${ }^{4}$ Department of Applied Physics, Ghent University - Belgium, EU \\ ${ }^{5}$ Asociasion EURATOM-CIEMAT para Fusion, CIEMAT - Madrid, Spain, EU \\ ${ }^{6}$ Consorzio RFX, ENEA-Euratom Association - Padua, Italy, EU \\ ${ }^{7}$ Associação EURATOM/IST, Centrode Fusão Nuclear - Lisbon, Portugal, EU \\ ${ }^{8}$ EFDA CSU - Boltzmannstrasse 2, 85748 Garching, Germany, EU \\ ${ }^{9}$ Association EURATOM-Tekes, VTT - P.O. Box 1000, FIN-02044 VTT, Finland, EU
}

received 27 March 2008; accepted in final form 20 May 2008

published online 16 June 2008

PACS 52.30.-q-Plasma dynamics and flow

\begin{abstract}
Results from recent measurements of carbon impurity ion toroidal and poloidal rotation velocities, ion temperature, ion density and the resulting radial electric field $\left(E_{r}\right)$ profiles are presented from an evolving Joint European Torus (JET) tokamak plasma over a range of energy and particle confinement regimes. Significant levels of edge plasma poloidal rotation velocity have been measured for the first time on JET, with maximum values of $\pm 9 \mathrm{~km} \mathrm{~s}^{-1}$. Such values of poloidal rotation provide an important contribution to the total edge plasma $E_{r}$ profiles. Large values of shear in the measured $E_{r}$ profiles are observed to arise as a consequence of the presence of the edge transport barrier (ETB) and do not appear to be necessary for their formation or destruction. These results have an important impact on potential mechanisms for transport barrier triggering and sustainment in present-day and future high-performance fusion plasmas.
\end{abstract}

Copyright (c) EPLA, 2008

Introduction. - The current operation scenario envisaged for the next step magnetic fusion device, ITER [1], is one with high energy and confinement times, the $\mathrm{H}$-mode. Despite large progress in the development of the $\mathrm{H}$-mode regime on tokamaks worldwide, an understanding of the triggering and sustainment mechanisms for the Low-to-High confinement regime (L-H) transition, various H-mode phases and the High-to-Low confinement phase $(\mathrm{H}-\mathrm{L})$ transitions remains elusive. A great deal of the theoretical and experimental research has concentrated on the role of the radial electric field, $E_{r}$, at the L-H transition, for example see $[1-5]$ and references therein. Large gradients in the edge $E_{r}$ are considered to lead to shear in the plasma $\mathbf{E} \times \mathbf{B}$ flow which effectively breaks up turbulent eddies and reduces cross-field transport. The edge transport barrier subsequently forms, leading to the improved plasma energy, particle and momentum

\footnotetext{
(a) See the appendix of Watkins M. L. et al., Fusion Energy 2006, Proceedings of the 21st International Conference, Chengdu, 2006 (IAEA) 2006.
}

confinement times characteristic of the H-mode. Experimental studies have shown that gradients in $E_{r}$ persist throughout $\mathrm{H}$-modes and are a general experimental feature of plasmas with an edge transport barrier. Results are presented in this paper from recent work to focus on this particular process, by considering the evolution of the measured impurity ion toroidal $\left(v_{\phi}\right)$ and poloidal $\left(v_{\theta}\right)$ rotation velocities and their contribution to the edge $E_{r}$, via the force balance equeation described below, over the changing phases of an H-mode plasma on the Joint European Torus (JET) tokamak.

Earlier experimental work in this area include very highly resolved measurements of $E_{r}$ at the plasma edge for a wide range of $\mathrm{H}$-mode discharges on DIII-D [2]. A welllike structure was observed in $E_{r}$ at the L-H transition in all plasmas. All the measured $E_{r}$ wells were negative with nearly the same spatial width, but differences in depth and under all conditions the shear in the $E_{r}$ profile persisted throughout the H-mode. The greatest contribution to $E_{r}$ came from changes in impurity poloidal rotation 
velocity, $v_{\theta}$, while the influence of the diamagnetic term only became appreciable tens of milliseconds into the $\mathrm{H}$-mode as the ion pressure increased. The sign of carbon ion $v_{\theta}$ was also observed to be consistently in the electron diamagnetic direction. More recent DIII-D measurements of $v_{\theta}$ profiles have been reported in [4] for Quiescent $\mathrm{H}$-modes. Charge Exchange Recombination Spectroscopy (CXRS) on carbon and neon impurity species, was used to measure the $E_{r}$ profile across the core and edge plasma. Following the application of velocity corrections due to the energy-dependent cross-sections and ion gyromotion, the experimental poloidal rotation profiles for both impurity species were found to be significantly greater than predictions of $v_{\theta}$. Previous JET studies by Hawkes et al. [6] have shown no evidence of changes in the impurity $v_{\theta}$ at the L-H transition, or during established $\mathrm{H}$-modes. Although this previous work demonstrates that the edge $E_{r}$ is strongly affected by the L-H transition, it is not clear whether the gradient in the $E_{r}$ profile is the cause or consequence of improved confinement in the $\mathrm{H}$-mode.

Recent enhancements to the JET edge plasma CXRS diagnostic have returned it to a balanced up-down symmetrical view of the neutral beam injection [7-9]. These changes together with new detectors allow accurate measurements of the impurity ion $v_{\theta}, v_{\phi}$, ion temperature, $T_{i}$, and ion density, $n$, profiles, with fine spatial resolution across the pedestal region. This letter presents the first detailed measurements of the evolution of the edge plasma $E_{r}$ profile and its contributing terms over all phases of a non-stationary JET H-mode plasma. The example plasma presented in this letter has been chosen since it covers many different phases of $\mathrm{L}$ - and $\mathrm{H}$-mode regimes and is a good representation of what is generally observed on JET with the improved diagnostic.

Experiment. - The evolution of the edge plasma rotation and $E_{r}$ is examined for pulse \# 69937, a single null configuration with the ion $\nabla B$ drift towards the magnetic null-point, $I_{p} / B_{t}=2.5 \mathrm{MA} / 2.7 \mathrm{~T}$ and $q_{95}=3.3$. This plasma had relatively high shaping and a divertor configuration such that the inner strikepoint remained on the inner vertical target, while the outer strikepoint location was on the outer horizontal target. The various H-mode transitions were accessed by slowly ramping the neutral beam power up and then back down at a rate of $1 \mathrm{MW} \mathrm{s}^{-1}$, as shown in fig. 1. A constant level of $1 \mathrm{MW}$ of Ion Cyclotron Resonance Heating ( $\mathrm{ICRH})$ power was applied throughout the beam heating phase. The L-mode plasma $n_{e}$ was controlled using active gas feedback on the deuterium fuelling, also shown in fig. 1, which led to variation of the level of gas puffing throughout the shot.

According to the force balance equation, $E_{r}$ can be written as

$$
E_{r}=\nabla P / n e Z+v_{\phi} B_{\theta}-v_{\theta} B_{\phi}
$$

where $P$ is the ion pressure, $Z$ is the ion charge number, $e$ is the electron charge, $n$ is the ion density, $v_{\phi}$ and $v_{\theta}$

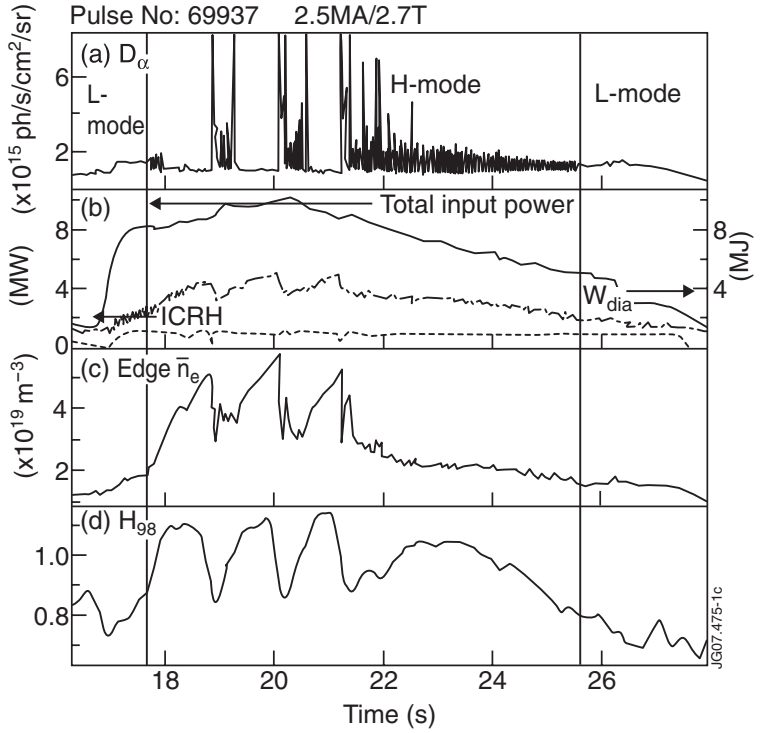

Fig. 1: General plasma parameters for shot \# 69937. (a) Divertor $D_{\alpha}$ signal, (b) total input power, ICRH power and plasma energy, (c) edge electron density, $\bar{n}_{e}$ and (d) H-mode confinement enhancement factor, $H_{98}$.

are the toroidal and poloidal velocities, respectively and $B_{\phi}$ and $B_{\theta}$ are the toroidal and poloidal magnetic fields, respectively. Equation (1) is valid for fuel and impurity ion species individually and in this paper all measurements were made with $\mathrm{C}^{6+}$ ions. It should be noted that the values and directions of $v_{\phi}$ and $v_{\theta}$ for different plasma ions can vary significantly from one another $[2-5]$. However, $E_{r}$ and the $\mathbf{E} \times \mathbf{B}$ velocity shear, apply to all plasma species.

The edge plasma viewing CXRS system on JET has views along fourteen radial channels locations ranging from mid-plane radius of, $R_{\text {mid }}=3.65-3.85 \mathrm{~m}(\rho \cong$ 0.65-1.2) with spatial resolution ranging from 1.2 to $2.3 \mathrm{~cm}$ and temporal resolution of $50 \mathrm{~ms}$. The balanced viewing geometry allows the measurements of $v_{\phi}$ and $v_{\theta}$ to be made self-consistently from line-of-sight velocities that have both toroidal and poloidal components. Further details on the diagnostic and its measurements can be found in [7].

The electron temperature, $T_{e}$, has been measured with a multichannel Electron Cyclotron Emission (ECE) radiometer with a time resolution of $1 \mathrm{~ms}$. The electron temperature at the top of the pedestal has been identified by the discontinuity in the gradient across the pedestal as defined in [10] and as used in previous JET studies [11-13]. The $T_{e}$ at the pedestal top at the $\mathrm{L}-\mathrm{H}$ transition is obtained by applying the pedestal position identified at the first clear $\mathrm{H}$-mode $T_{e}$ pedestal, to the $T_{e}$ profile at the time of the transition. The line integrated edge $n_{e}$ is measured with an interferometer along a single chord at $R_{\text {mid }}=3.74 \mathrm{~m}$ for this shot. The line average edge $\bar{n}_{e}$ has been calculated by dividing the measured line integrated $n_{e}$ by the chord length in the plasma.

Results. - The L-H transition in this shot occurs at $t=17.7 \mathrm{~s}$ and is characterised by the start of very small, 


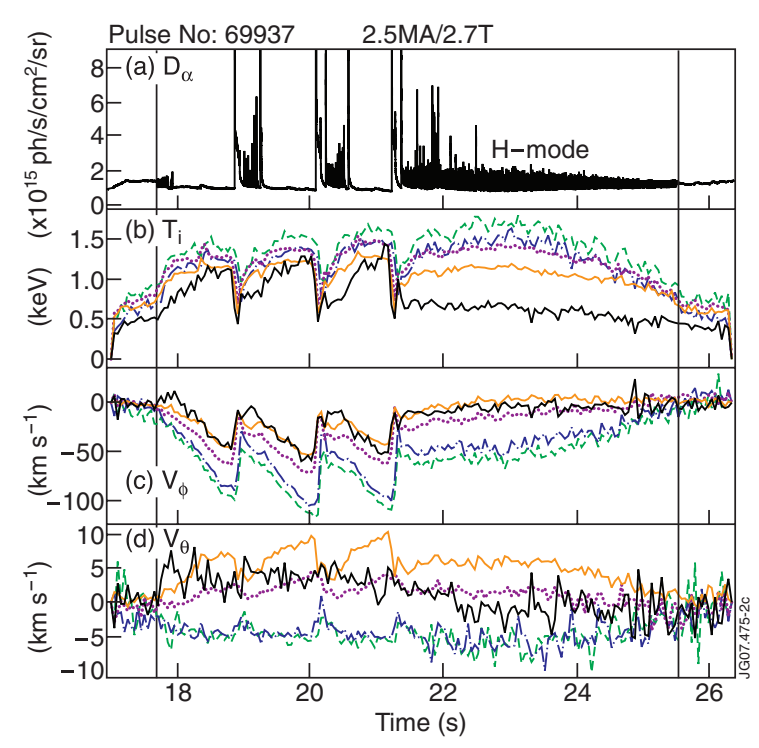

Fig. 2: Overview of the $T_{i}, v_{\phi}$ and $v_{\theta}$ across the L-mode and $\mathrm{H}$-mode phases of shot \# 69937 at $I_{p} / B_{t}=2.5 \mathrm{MA} / 2.7 \mathrm{~T}$. The colour of each time trace corresponds to the radial location indicated in fig. 3 by corresponding vertical lines of the same colour.

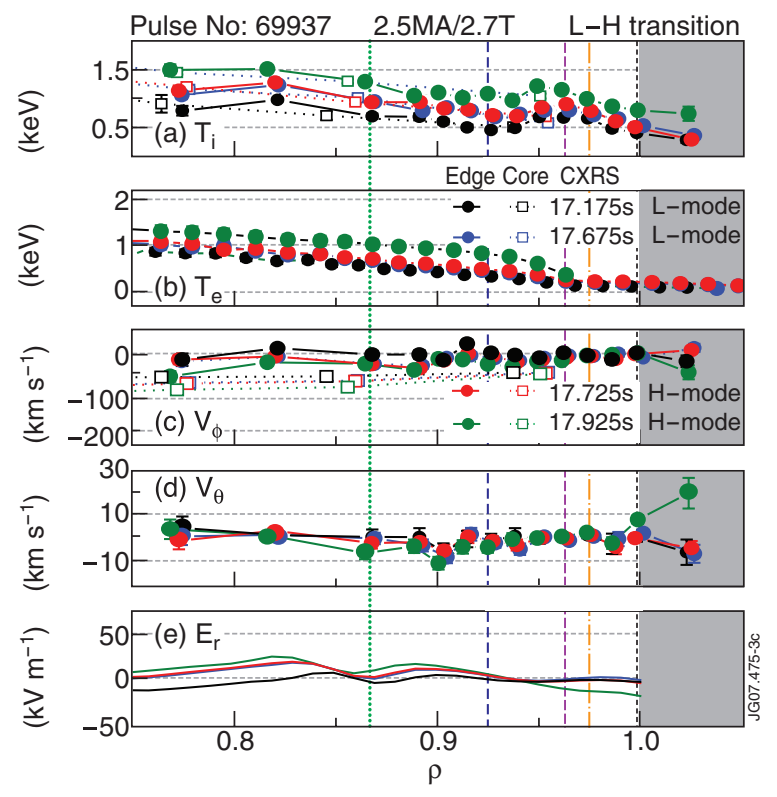

Fig. 3: Radial profiles for the edge plasma (a) $T_{i}$, (b) $T_{e}$, (c) $v_{\phi}$ and (d) $v_{\theta}$ and (e) $E_{r}$ values across the L-H transition for shot \# 69937. The vertical colour lines indicate the radial locations of the time traces in corresponding colours in figs. 2 and 4 .

irregular events which degrade the edge transport barrier, known as edge localised modes (ELMs). The L-H transition is also accompanied by increased values of plasma $\bar{n}_{e}$, the plasma energy, $W_{d i a}$ and the plasma confinement enhancement factor, $H_{98}$ [14], indicated by the first vertical line in figs. 1 and 2. The threshold power for access to the $\mathrm{H}$-mode in this plasma was $P_{t h}=6.8 \mathrm{MW}$ at an edge density of $\bar{n}_{e}=1.8 \times 10^{19} \mathrm{~m}^{-3}$. The confinement

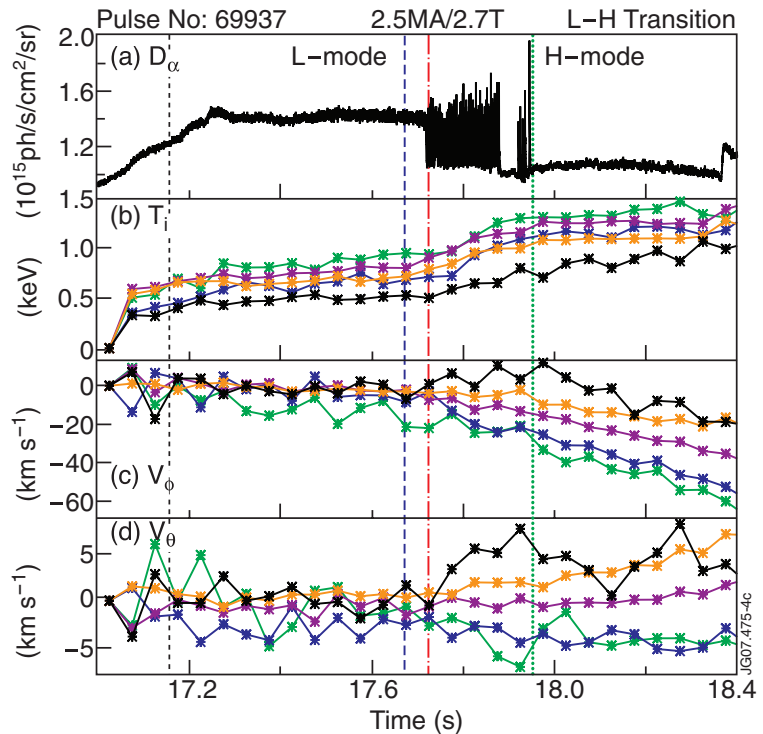

Fig. 4: Time traces showing (a) the divertor $D_{\alpha}$ signal, (b) the edge $T_{i}$, (c) edge $v_{\phi}$ and (d) edge $v_{\theta}$ values for radial locations indicated in fig. 3, across the L-H transition for shot \# 69937.

factor directly following the L-H transition, was $H_{98}=0.9$ and it increased to 1.0 by the end of the first short, ELMy phase. The values of $T_{i}, v_{\phi}$ and $v_{\theta}$ at radial locations indicated in fig. 3 by vertical, colour lines are plotted in their corresponding colours as a function of time in fig. 2 and in more detail in fig. 4 along with the divertor $D_{\alpha}$ signal. This method of profile and time trace cross-correlation is used throughout the letter. The radial profiles of the edge plasma $T_{i}, T_{e}, v_{\phi}, v_{\theta}$ and $E_{r}$ are plotted as a function radius normalised to the plasma radius in fig. 3 for time-slices in the L-mode phase, across the L-H transition and following the initial ELMy phase, all indicated by the corresponding vertical, colour lines in fig. 4. Measurements of $T_{i}$ and $v_{\phi}$ across the core plasma are also included in the profile plots as open symbols. During the L-mode phase (black and blue profiles in fig. 3) a very small increase is observed in the $T_{i}$ and $T_{e}$ profiles, with the pedestal top values of $T_{i}=790( \pm 22) \mathrm{eV}$ at $\rho=0.95$ and $T_{e}=320( \pm 32) \mathrm{eV}$ at $\rho=0.94$ for the last L-mode time-slice. A much more significant increase in the temperature pedestals can be seen in figs. $3(\mathrm{a}, \mathrm{b})$ and 4(b) following the transition to H-mode. The edge plasma $v_{\theta}$ profile remains close to zero throughout the L-mode phase as shown by the profiles in fig. 3(d) and the corresponding time traces in figs. 2(d) and 4(d). However, $v_{\theta}$ is observed to spin-up along the outermost two channels (green profile) after the $\mathrm{L}-\mathrm{H}$ transition, to a maximum value of $8( \pm 2) \mathrm{km} \mathrm{s}^{-1}$ just within the last closed magnetic flux surface (LCFS). The edge $v_{\phi}$ profile also remains very flat at around $0 \mathrm{~km} \mathrm{~s}^{-1}$ during the L-mode phase as shown in figs. 2(c), 3(c) and 4(c). At the start of the $\mathrm{H}$-mode, however, the innermost channels measure a strong increase in $v_{\phi}$ in the negative co-current direction (blue time traces), while just within the LCFS the 


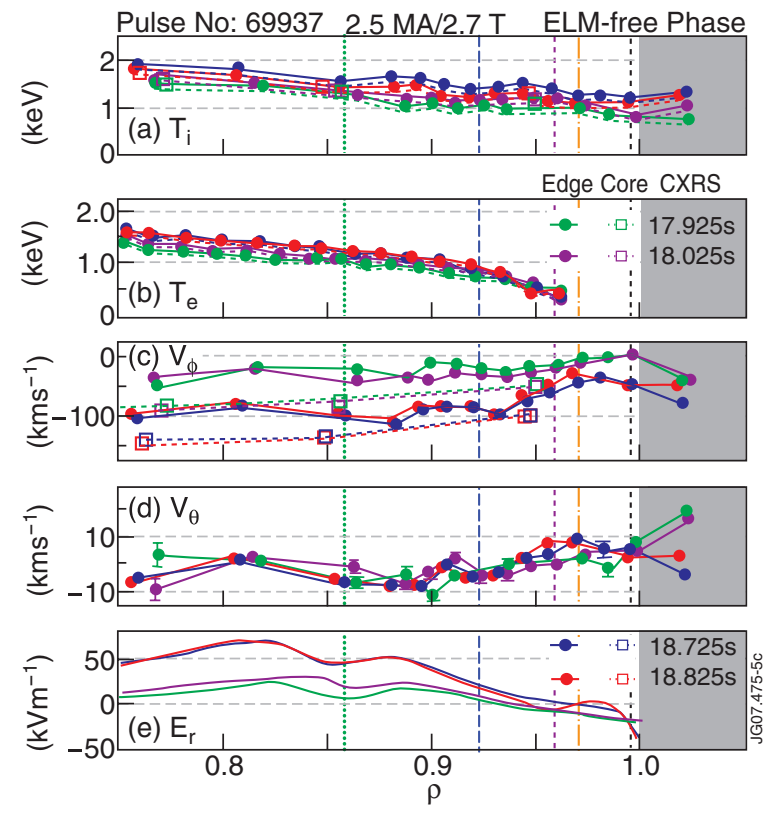

Fig. 5: Radial profiles for the time-slices indicated by vertical lines in fig. 6 in corresponding colours, in the ELM-free phase of the H-mode for shot \# 69937.

plasma briefly spins up to $12( \pm 5) \mathrm{km} \mathrm{s}^{-1}$ in the positive counter-current direction (black time-trace). Following the transition to the ELM-free phase all channels observe toroidal velocity spin-up in the co-current direction and the development of a gradient in $v_{\theta}$. The corresponding $E_{r}$ profiles, fig. $3(\mathrm{e})$, remain flat and close to $0 \mathrm{kV} \mathrm{m}^{-1}$ across the edge plasma in the L-mode phase. A gradient in $E_{r}$ subsequently develops in the $\mathrm{H}$-mode with a minimum value of $-20( \pm 6) \mathrm{kV} \mathrm{m}^{-1}$ at the LCFS, green profile in fig. $3(\mathrm{~d})$ at $t=17.925 \mathrm{~s}$, with the $v_{\phi}$ component providing $65 \%$ of the value of the minimum in $E_{r}$.

On entering the ELM-free phase the plasma edge $\bar{n}_{e}$ continues to increase at a rate similar to that during the initial ELMy phase and the value of $H_{98}$ increases from 1.0 to a maximum of 1.1 at $18.4 \mathrm{~s}$, as seen in fig. 1 . Both the $T_{e}$ and $T_{i}$ pedestals continue to develop during the ELMfree $\mathrm{H}$-mode, with the top of pedestals reaching maximum values of $T_{i}=1500( \pm 15) \mathrm{eV}$ and $T_{e}=870( \pm 87) \mathrm{eV}$ both at $\rho=0.94$ (blue profiles), shown in figs. 5 and 6 . The $v_{\theta}$ profile demonstrates a great deal of evolution during the ELM-free phase, with the development of a sheared region of rotation across the $T_{i}$ pedestal, as shown in fig. $5(\mathrm{~d})$. Just within the LCFS, $v_{\theta}$ decreases to around $2 \mathrm{~km} \mathrm{~s}^{-1}$ as the $T_{i}$ gradient region moves further in towards the core plasma, as shown by the green and red profiles in fig. 5(d) and by the black time-trace in fig. $6(\mathrm{~d})$. Over the same time period the channels closest to the pedestal top measure a gradual increase in $v_{\theta}$ to a maximum of $7.0( \pm 1) \mathrm{km} \mathrm{s}^{-1}$ in the electron diamagnetic direction, see fig. 5(d) and red trace in fig. 6(d). At the same time, the innermost channel shown, see green and blue time traces in fig. 6(d), increases to a maximum

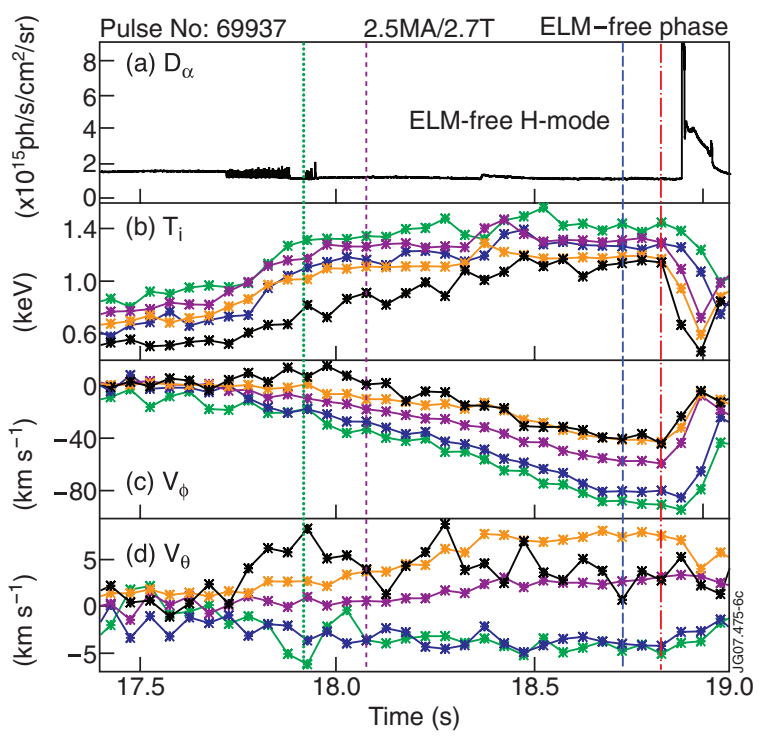

Fig. 6: Time traces across the ELM-free phase of the H-mode for shot \# 69937 for (a) the divertor $D_{\alpha}$, (b) edge $T_{i}$, (c) edge $v_{\phi}$ and $(\mathrm{d})$ edge $v_{\theta}$ for corresponding radii indicated by vertical lines in fig. 5 .

of $-6( \pm 2) \mathrm{km} \mathrm{s}^{-1}$ in the ion diamagnetic direction. As shown by the red profile in fig. $5(\mathrm{~d})$ the overall effect is the development of a well-like structure in the $v_{\theta}$ profile across the edge plasma. The $v_{\phi}$ profile continues to develop during the ELM-free phase with spin-up in the co-current direction. While the region close to LCFS demonstrates relatively little increase in $v_{\phi}$, the inner channels measure an increase from $-9.6( \pm 7) \mathrm{km} \mathrm{s}^{-1}$ to $-88( \pm 4) \mathrm{km} \mathrm{s}^{-1}$ at $9 \mathrm{~cm}$ from the LCFS, as seen in the red and blue profiles of fig. 5 . The outermost channels on the other hand measure a small increase of $29( \pm 3) \mathrm{km} \mathrm{s}^{-1}$ over this time, resulting in steepening of the edge $v_{\phi}$ gradient. The profiles of $E_{r}$ over this phase become more positive as $v_{\theta}$ profile increases across the edge plasma. The result is a steeper $E_{r}$ gradient in the edge region, with the pressure and $v_{\theta}$ terms provide similar contributions in the pedestal region.

The first of three very large ELMs occurs at $t=18.9 \mathrm{~s}$. The $T_{i}, T_{e}, v_{\phi}, v_{\theta}$ and $E_{r}$ profiles across the second ELM are plotted in fig. 7 and the corresponding time-traces over all three ELMs are shown in fig. 8, for the corresponding radial locations. The three large ELMs were characterised by decreases in plasma energy 1.1 MJ, 1.4 MJ and 1.2 MJ, respectively. Comparing the $T_{i}$ profiles prior to the second large ELM (black and red profiles) with that directly after (blue profile), a decrease in $T_{i}$ is first observed within $4 \mathrm{~cm}$ of the LCFS, while the inner region maintains pre-ELM values. In the subsequent time-slice $50 \mathrm{~ms}$ later, the inner channels also measure a decrease in $T_{i}$ (green profile) and the overall decrease in $T_{i}$ is from $1373( \pm 28) \mathrm{eV}$ at $20.075 \mathrm{~s}$ to $908( \pm 13.2) \mathrm{eV}$ at $20.175 \mathrm{~s}$ at the pedestal top. As shown in fig. 7 (b) the pedestal $T_{e}$ also falls from $860( \pm 86) \mathrm{eV}$ to a minimum of $200( \pm 20) \mathrm{eV}$ following the ELM. These 


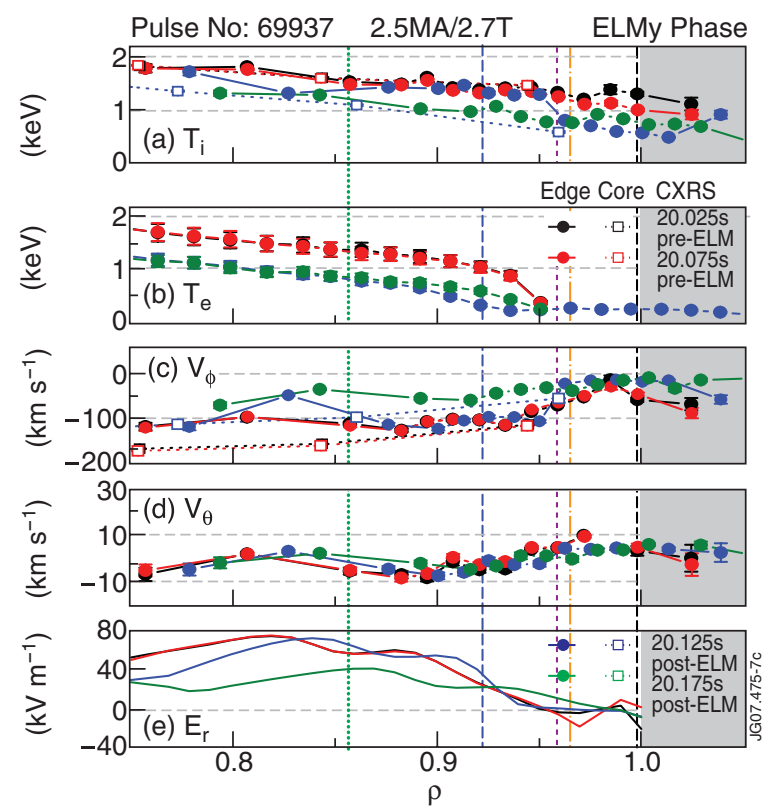

Fig. 7: Radial profiles of (a) $T_{i}$, (b) $T_{e}$, (c) $v_{\phi}$, (d) $v_{\theta}$ and (e) $E_{r}$ before and following a large ELM indicated in fig. 8, for shot \# 69937.

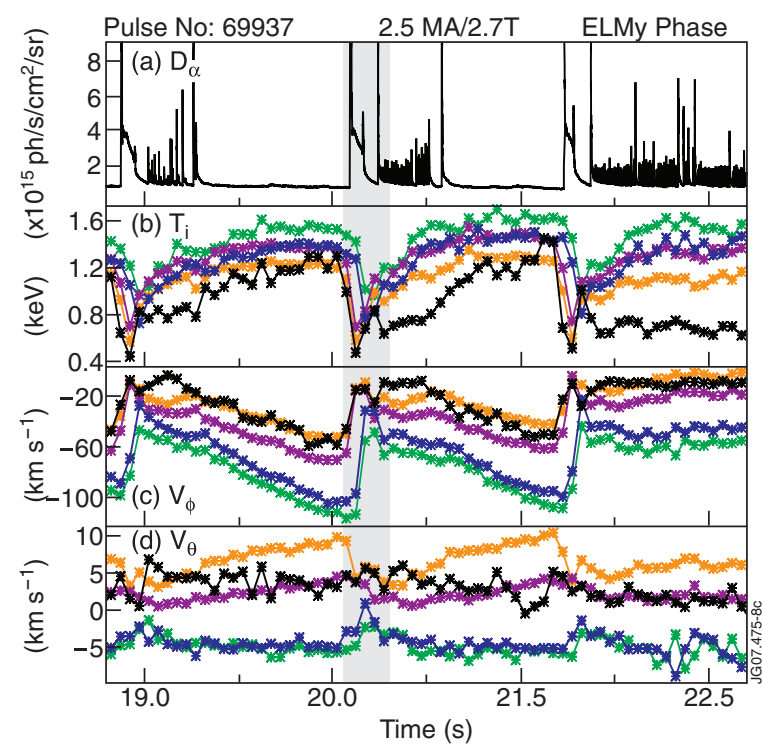

Fig. 8: Time traces across the large ELMs phase of the H-mode for shot \# 69937.

large ELMs also have a significant effect on the $v_{\phi}$ rotation profile, shown in fig. $7(\mathrm{c})$, with the region $4 \mathrm{~cm}$ inside the LCFS the first to demonstrate a decrease in $v_{\phi}$ by a maximum of $56 \mathrm{~km} \mathrm{~s}^{-1}$, (blue profile). In the following time-slice (green profile) the remainder of the edge $v_{\phi}$ profile is also reduced with the mitigation of the edge $v_{\phi}$ gradient. The $v_{\theta}$ time traces also demonstrate a slowing down following the ELMs, with the most sensitive channels within the $T_{i}$ gradient region and can be seen in figs. 2(d) and $8(\mathrm{~d})$ to drop by $5( \pm 0.5) \mathrm{km} \mathrm{s}^{-1}$ in $v_{\theta}$ over each large ELM. Despite these changes the overall $v_{\theta}$ profile is largely
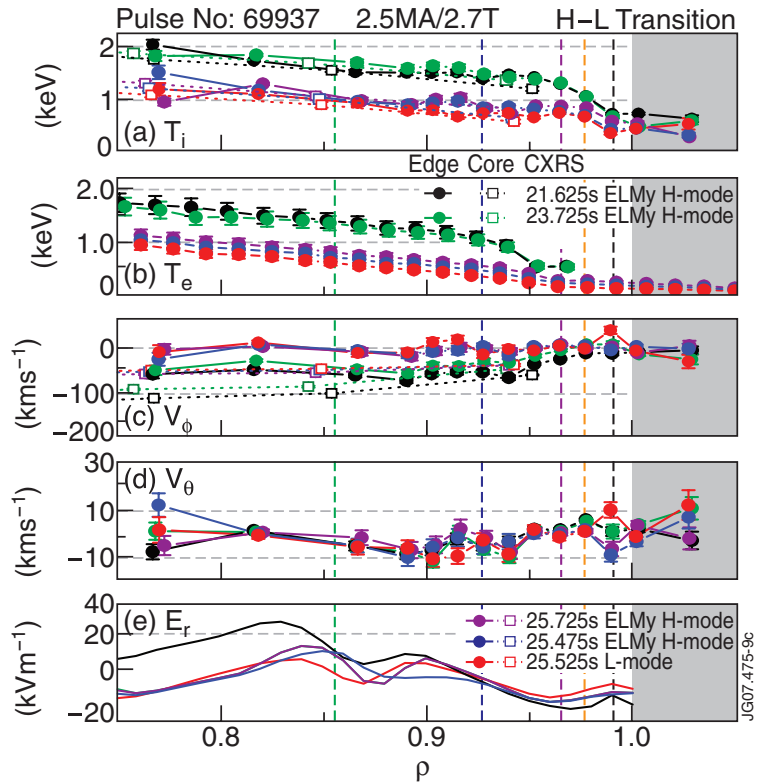

Fig. 9: Radial profiles at the start of the high frequency ELM phase and across the H-L transition for shot \# 69937, for times indicated by vertical colour lines in fig. 10 .

unaffected by the ELMs. The corresponding profiles of $E_{r}$ shown in fig. $7(\mathrm{e})$, show a degradation of the pedestal $E_{r}$ gradient following the ELM, compare the red with green profiles. The main source of the reduction in $E_{r}$ is from the $40 \%$ decrease of the $v_{\phi}$ term in the pedestal region following the ELM.

The input power ramp down starts at $20.4 \mathrm{~s}$ and the third large ELM is directly followed by a phase of highfrequency small ELMs, as shown in figs. 1, 2 and 10. The $\mathrm{H}$-mode remains in this high-frequency ELMy phase until the transition to L-mode at $25.5 \mathrm{~s}$. The power threshold for the $\mathrm{H}-\mathrm{L}$ transition in this shot was $P_{H L}=5.4 \mathrm{MW}$ at an edge $\bar{n}_{e}=1.7 \times 10^{19} \mathrm{~m}^{-3}$. The confinement factor at the start of the high-frequency ELMs falls to a minimum of $H_{98}=0.9$ at $21.4 \mathrm{~s}$ and subsequently recovers to a maximum value of $H_{98}=1.1$ at $22.71 \mathrm{~s}$, as shown in fig. 1 . The confinement factor remains at this value until $23.62 \mathrm{~s}$ after which it gradually falls reaching a value of $H_{98}=1$ at the H-L transition. Comparing figs. 7 (red profile) with fig. 9 (black and green profiles) the effect of the decrease in power and the onset of high-frequency ELMs is first seen in the outermost, edge plasma, closest to the LCFS as a clear reduction in $T_{i}$. As the input power decreases further, the ELM frequency increases further, the rest of the $T_{i}$ pedestal is also modified, with the pedestal $T_{i}$ falling from $1310( \pm 20) \mathrm{eV}$ at $\rho=0.95$ to $700( \pm 17) \mathrm{eV}$ (blue profile) at $\rho=0.95$, the $T_{i}$ barrier width shrinks from $3 \mathrm{~cm}$ to $2 \mathrm{~cm}$. A similar change in $T_{e}$ pedestal is observed with a drop in the pedestal $T_{e}$ from $906( \pm 91) \mathrm{eV}$ to $423( \pm 42) \mathrm{eV}$. The $v_{\theta}$ profiles demonstrate a general erosion of the well-like structure in the profile as the power ramps down, with a reduction of difference in $v_{\theta}$ to a minimum value of $8( \pm 3) \mathrm{km} \mathrm{s}^{-1}$ at $25.48 \mathrm{~s}$ preceding the H-L transition. The 


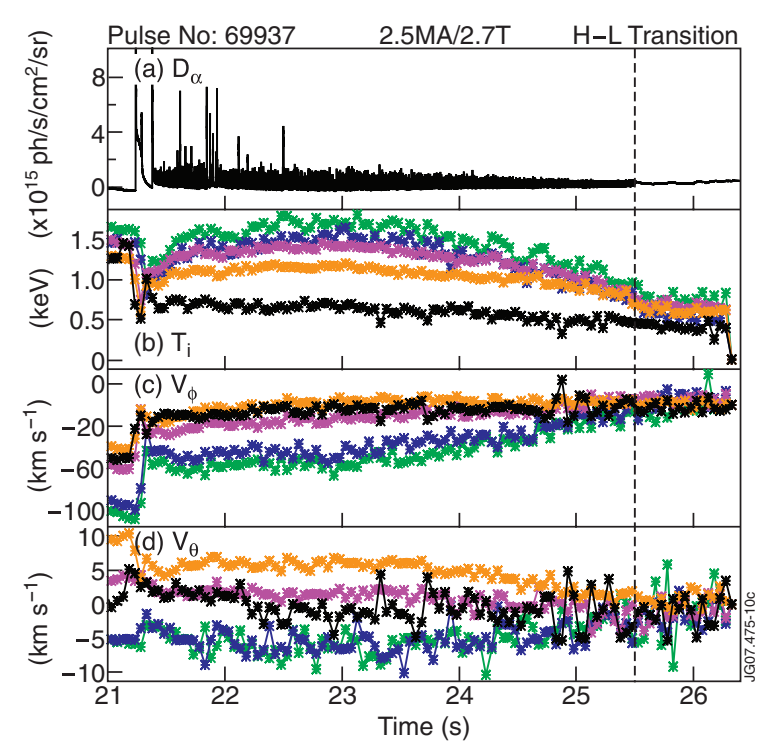

Fig. 10: Time traces across the high frequency ELM phase and the H-L transition of shot \# 69937 for (a) the divertor $D_{\alpha}$, (b) $T_{i}$, (c) $v_{\phi}$ and (d) $v_{\theta}$, for radial channels indicated in fig. 9 by vertical lines in corresponding colours.

loss of shear in $v_{\theta} 300 \mathrm{~ms}$ before the $\mathrm{H}-\mathrm{L}$ transition can also be clearly seen in the time-traces shown in fig. 10. Finally, the edge $v_{\phi}$ evolves to a very flat profile at around $0 \mathrm{~km} \mathrm{~s}^{-1}$ by the end of the ELMy H-mode also shown in figs. 9 and 10. The degradation of all three terms over the power ramp down contribute approximately equally to the reduction and flattening off of the $E_{r}$ profile towards the end of the ELMy phase as shown by the profiles in fig. 9(e).

Conclusions. - Highly spatially resolved measurements of the edge plasma, carbon ion $T_{i}, T_{e}, v_{\theta}, v_{\phi}$ and $E_{r}$ have been documented for the first time through many different stages of a JET L- and H-mode plasma. No evidence of spin-up of $v_{\theta}$ prior to the L-H transition has been observed with a time resolution of $50 \mathrm{~ms}$, although a clear increase in $v_{\theta}$ is recorded directly following the transition to H-mode. This localised spin-up precedes the onset of an ELM-free phase in which further development of the $T_{i}, T_{e}, v_{\phi}$ and $v_{\theta}$ profile structure is clearly seen. The $E_{r}$ profile remains very flat throughout the L-mode phase and strong edge gradients only start to form during the ELM-free H-mode. The very large ELMs that follow the ELM-free phase have a significant impact on the $T_{i}$ and $v_{\phi}$ profiles, eroding the pedestal gradients. Pedestal region $v_{\theta}$ slows down by up to $5 \mathrm{~km} \mathrm{~s}^{-1}$ following the large ELMs. Finally, the $E_{r}$ profile has also been examined on JET over the H-L transition. The pedestal $T_{i}, T_{e}, v_{\phi}$ and $v_{\theta}$ all demonstrate a gradual relaxation of their gradients as the input power is ramped down and the disappearance of the edge transport barrier is preceded by the erosion any significant $v_{\theta}$ shear. These data suggest that the development of significant shear in $E_{r}$ arises as a consequence of the high confinement phase of the plasma and is not required to enter or maintain the $\mathrm{H}$-mode on JET. This important result indicates that $\mathbf{E} \times \mathbf{B}$ shear suppression of turbulence does not trigger the transport barrier formation, although it may well play a role in transport barrier sustainment and dynamics.

$$
* * *
$$

This work was carried out within the framework of EFDA and was partly funded by the UK EPSRC and EC under the contract of Association between EURATOM/UKAEA. The views and opinions expressed herein do not necessarily reflect those of the European Commission.

\section{(c)Euratom, 2008.}

\section{REFERENCES}

[1] Ikeda K. et al., Nucl. Fusion, 47 (2007) S18.

[2] Gohil P., Burrell K. H. and Carlstrom T. N., Nucl. Fusion, 38 (1998) 93.

[3] Testa D., Garzotti L. and Giroud C., Nucl. Fusion, 46 (2006) 562.

[4] Solomon W. M., Burrell K. H., Andre R., Baylor L. R., Budney R., Gohil P., Groebner R. J., Holcomb C. T., Houllberg W. A. and Wade M. R., Phys. Plasmas, 13 (2006) 056116.

[5] Kim J. et al., Phys. Rev. Lett., 72 (1994) 2199.

[6] Hawkes N. C. et al., Plasma Phys. Control. Fusion, 38 (1996) 1261.

[7] Andrew Y., Hawkes N. C. and Crombe K., Rev. Sci. Instrum., 77 (2006) 10E913.

[8] Hawkes N. C. and Peacock N., Rev. Sci. Instrum., 63 (1992) 5164.

[9] Hawkes N. C., Rev. Sci. Instrum., 68 (1997) 2051.

[10] Breger P., Flewin C., Zastrow K.-D., Davies S. J., Hawkes N. C., Konig R. W. T., Pietrzyk Z. A., Porte L., Summers D. D. R. and Von Hellerman M. G., Plasma Phys. Control. Fusion, 40 (1998) 347.

[11] Righi E. et al., Nucl. Fusion, 39 (1999) 309.

[12] Andrew Y., Hawkes N. C., O'Mullane M. G., Sartori R., Beurskens M. N. A., Coffey I., Joffrin E., Loarte A., McDonald D. C., Prentice R., Saibene G., Suttrop W. and Zastrow K.-D., Plasma Phys. Control. Fusion, 46 (2004) 337.

[13] Andrew Y., Sartori R., Righi E., de la Luna E., Hacquin S., Howell D., Hawkes N. C., Horton L. D., Huber A., Korotkov A. and O'Mullane M. G., Plasma Phys. Control. Fusion, 48 (2006) 479.

[14] ITER Physics Expert Groups on CDBM, Nucl. Fusion, 39 (1999) 2175. 